PROCEEDINGS OF THE

AMERICAN MATHEMATICAL SOCIETY

Volume 137, Number 6, June 2009, Pages 1989-1996

S 0002-9939(09)09827-X

Article electronically published on January 29, 2009

\title{
BORG-TYPE UNIQUENESS THEOREMS FOR PERIODIC JACOBI OPERATORS WITH MATRIX-VALUED COEFFICIENTS
}

\author{
EVGENY KOROTYAEV AND ANTON KUTSENKO
}

(Communicated by Peter A. Clarkson)

\begin{abstract}
We give a simple proof of Borg-type uniqueness theorems for periodic Jacobi operators with matrix-valued coefficients.
\end{abstract}

\section{INTRODUCTION}

Consider a self-adjoint Jacobi operator $J$ acting on $\ell^{2}(\mathbb{Z})^{m}, m \geqslant 1$, and given by (1.1)

$(J y)_{n}=a_{n} y_{n+1}+b_{n} y_{n}+a_{n-1}^{*} y_{n-1}, \quad n \in \mathbb{Z}, \quad y=\left(y_{n}\right)_{n \in \mathbb{Z}} \in \ell^{2}(\mathbb{Z})^{m}, \quad y_{n} \in \mathbb{C}^{m}$,

where $\operatorname{det} a_{n} \neq 0$ and $a_{n}, b_{n}=b_{n}^{*}, n \in \mathbb{Z}$ are $p$-periodic sequences of the complex $m \times$ $m$ matrices. We denote the class of such operators by $\mathfrak{J}_{p}$. It is well known that the spectrum $\sigma(J)$ of $J$ is absolutely continuous and consists of non-degenerate intervals $\left[\lambda_{n-1}^{+}, \lambda_{n}^{-}\right], \lambda_{n-1}^{+}<\lambda_{n}^{-} \leqslant \lambda_{n}^{+}, n=1, \ldots, N<\infty$. These intervals are separated by the gaps $\gamma_{n}=\left(\lambda_{n}^{-}, \lambda_{n}^{+}\right), n=1, \ldots, N-1$ with the length $>0$. Introduce the fundamental $m \times m$ matrix-valued solutions $\varphi=\left(\varphi_{n}(z)\right)_{n \in \mathbb{Z}}, \vartheta=\left(\vartheta_{n}(z)\right)_{n \in \mathbb{Z}}$ of the equation

$a_{n} y_{n+1}+b_{n} y_{n}+a_{n-1}^{*} y_{n-1}=z y_{n}, \quad \varphi_{0} \equiv \vartheta_{1} \equiv 0, \varphi_{1} \equiv \vartheta_{0} \equiv I_{m}, \quad(z, n) \in \mathbb{C} \times \mathbb{Z}$,

where $I_{m}$ is the identity $m \times m$ matrix. We define the $2 m \times 2 m$ monodromy matrix $M_{p}$ by

$$
M_{p}(z)=\left(\begin{array}{cc}
\vartheta_{p}(z) & \varphi_{p}(z) \\
\vartheta_{p+1}(z) & \varphi_{p+1}(z)
\end{array}\right), \quad z \in \mathbb{C} .
$$

Let $\tau_{1}(z), \ldots, \tau_{2 m}(z)$ be eigenvalues of $M_{p}(z)$. Recall the well-known fact $\sigma(J)=$ $\bigcup_{j=1}^{2 m}\left\{z \in \mathbb{C}:\left|\tau_{j}(z)\right|=1\right\}$; see $\left[\mathrm{KKu}\right.$. Let $J^{0}$ be the unperturbed Jacobi matrix with $a_{n}^{0}=I_{m}, b_{n}^{0}=0$ and $\tau_{j}^{0}$ be the corresponding eigenvalues. Note that $\sigma\left(J^{0}\right)=$ $[-2,2]$ and $\left|\tau_{j}^{0}(z)\right|=1$ for all $(z, j) \in \sigma\left(J^{0}\right) \times \mathbb{N}_{2 m}$, where $\mathbb{N}_{s}=\{1, \ldots, s\}$. Introduce

Received by the editors January 23, 2008.

2000 Mathematics Subject Classification. Primary 47B39, 34A55, 47B36.

(C)2009 American Mathematical Society Reverts to public domain 28 years from publication 
the following classes of Jacobi operators:

$$
\operatorname{Iso}_{p}\left(J^{0}\right)=\left\{J \in \mathfrak{J}_{p}: \sigma(J)=[-2,2], \quad\left|\tau_{j}(z)\right|=1 \text {, for all }(z, j) \in \sigma(J) \times \mathbb{N}_{2 m}\right\},
$$

$$
\operatorname{Uni}_{p}\left(J^{0}\right)=\left\{J \in \mathfrak{J}_{p}: \quad J \text { is unitary equivalent to } J^{0}\right\},
$$

$$
\mathfrak{J}_{p}^{0}=\left\{J \in \mathfrak{J}_{p}: a_{n} a_{n}^{*}=I_{m}, b_{n}=0 \text { for all } n \in \mathbb{Z}\right\} .
$$

We now formulate our first theorem.

Theorem 1.1. i) The following identities hold true:

$$
\operatorname{Iso}_{p}\left(J^{0}\right)=\operatorname{Uni}_{p}\left(J^{0}\right)=\mathfrak{J}_{p}^{0} .
$$

ii) Let $J \in \operatorname{Iso}_{p}\left(J^{0}\right)$ and let each $a_{n}, n \in \mathbb{Z}$ have only positive eigenvalues. Then $J=J^{0}$.

Case ii) includes two cases: (1) $a_{n}=a_{n}^{*}>0$; (2) $a_{n}$ are lower triangular matrices with real positive entries on the diagonal and $J$ is a so-called $(2 m+1)$-band matrix.

The Borg-type uniqueness Theorem 1.1(ii) was proved in [CGR] for the partial case $a_{n}>0, n \in \mathbb{Z}$. In their proof the authors obtained the Herglotz formula for the Green function of the Jacobi matrix. Then using corresponding asymptotics at high energy, they determined the trace formulas, which were used to prove the Borg-type theorem for periodic Jacobi matrices. We give a simple proof of Theorem 1.1 based on our trace formula (2.1) and the sharp estimate (2.5). The Borg-type result in the scalar case $m=1$ was first settled by Flaschka $[\mathrm{F}$. There is an enormous literature on inverse spectral problems for scalar (i.e., $m=1$ ) periodic Jacobi matrices (see BGGK], K], KKu1, KKu2, VM], book T] and the references therein), but very little for matrix-valued periodic Jacobi operators (see $\mathrm{CGR}$, $\mathrm{KKu}$ and the references therein). Note that the complete solution of the inverse problem for finite matrix-valued Jacobi operators was obtained recently [BCK].

Recall the standard fact about the direct integrals for the operators with periodic coefficients; see [RS. The operator $J$ is unitarily equivalent to the operator $\widehat{J}=$ $\int_{[0,2 \pi)}^{\oplus} K_{p}\left(e^{i x}\right) \frac{d x}{2 \pi}$ acting in $\int_{[0,2 \pi)}^{\oplus} \mathcal{H} \frac{d x}{2 \pi}$, where $\mathcal{H}=\mathbb{C}^{p m}$ and the $p m \times p m$ matrix $K_{p}(\tau)$ is given by

$$
K_{p}(\tau)=\left(\begin{array}{ccccc}
b_{1} & a_{1} & 0 & \ldots & \tau^{-1} a_{p} \\
a_{1}^{*} & b_{2} & a_{2} & \ldots & 0 \\
0 & a_{2}^{*} & b_{3} & \ldots & 0 \\
\ldots & \ldots & \ldots & \ldots & \ldots \\
\tau a_{p}^{*} & 0 & \ldots & a_{p-1}^{*} & b_{p}
\end{array}\right), \quad \tau \in \mathbb{S}^{1}=\{\tau:|\tau|=1\} ;
$$

e.g., see [KKu]. Let $\left(\lambda_{n}(\tau)\right)_{n=1}^{p m}$ be eigenvalues of $K_{p}(\tau)$. In $[\mathrm{KKu}$ we obtained the spectral data (finite number of eigenvalues of $K_{p}(\tau)$ ), which determine uniquely all multipliers $\tau_{j}(\cdot), j=1, \ldots, 2 m$ and the spectrum of $J$ including the multiplicity. In our main Theorem 1.2 we give the finite spectral data which are necessary and sufficient for $J=J^{0}$. Let $c=\operatorname{det} \prod_{n=1}^{p} a_{n}$.

Theorem 1.2. Let $a_{n}>0, b_{n}=b_{n}^{*}$ be real $m \times m$ matrices for all $n \in \mathbb{Z}$.

i) Let $c=1$. Then $\sum_{n=1}^{m p} \lambda_{n}^{2}(\tau)=2 p m$ for some $\tau \in \mathbb{S}^{1}$ iff $J=J^{0}$.

ii) Let $c=1$ and let $\varkappa_{1} \in \mathbb{R}$. Then the eigenvalues have the form $\lambda_{s}\left(e^{i \varkappa_{1}}\right)=$ $2 \cos \frac{\varkappa_{1}+2 \pi(s-1)}{p}$ for all $s \in \mathbb{N}_{m p}$ iff $J=J^{0}$. 
iii) Let $\varkappa_{1}, \varkappa_{2} \in \mathbb{R}$ and $\cos \varkappa_{1} \neq \cos \varkappa_{2}$. Then the eigenvalues have the form $\lambda_{s}\left(e^{i \varkappa_{1}}\right)=2 \cos \frac{\varkappa_{1}+2 \pi(s-1)}{p}$ for all $s \in \mathbb{N}_{m p}$ and $\lambda_{n}\left(e^{i \varkappa_{2}}\right)=2 \cos \frac{\varkappa_{2}+2 \pi n_{1}}{p}$ for all $n \in \mathbb{N}_{m}$, for some $n_{1} \in \mathbb{N}_{p}$ and $e^{i 2 \varkappa_{2}} \neq 1$ (if $e^{i 2 \varkappa_{2}}=1$, then additional eigenvalues $\lambda_{n+m}\left(e^{i \varkappa_{2}}\right)=\lambda_{1}\left(e^{i \varkappa_{2}}\right) \neq \pm 2$ for all $\left.n \in \mathbb{N}_{m}\right)$ iff $J=J^{0}$.

Remark. 1) Note that the condition $e^{i 2 \varkappa_{2}} \neq 1$ in iii) is associated with the unperturbed operator $J^{0}$, where the endpoints of the spectrum $\sigma\left(J^{0}\right)=[-2,2]$ have the multiplicity $m$, as the zeros of the determinant $D_{p}(z, \pm 1)$, where $D_{p}(z, \tau)=$ $\operatorname{det}\left(M_{p}(z)-\tau I_{2 m}\right)$. Each point from $(-2,2)$ has multiplicity $2 m$ as the zero of the determinant $D_{p}(z, \tau), \tau \neq \pm 1$.

2) Consider the case $m=2$ and $\varkappa_{1}=0, \varkappa_{2}=\pi$. Let periodic eigenvalues $\lambda_{s}(1)=2 \cos \frac{2 \pi(s-1)}{p}$ for all $s \in \mathbb{N}_{2 p}$ and let anti-periodic eigenvalues $\lambda_{s}(-1)=$ $2 \cos \frac{\pi(2 n+1)}{p} \neq \pm 2$ for all $s \in \mathbb{N}_{4}$ and for some $n \in \mathbb{N}_{p}$. Then we deduce that $J=J^{0}$.

\section{Proof of Theorems 1.1 and 1.2}

We need the following results. Recall that $c=\operatorname{det} \prod_{1}^{p} a_{n}$.

Lemma 2.1. i) For any $\tau \in \mathbb{S}^{1}$ the following identities and estimate are fulfilled:

$$
\begin{gathered}
\operatorname{Tr} K_{p}(\tau)=\sum_{n=1}^{m p} \lambda_{n}(\tau)=\sum_{n=1}^{p} \operatorname{Tr} b_{n}, \operatorname{Tr}\left(K_{p}(\tau)\right)^{2}=\sum_{n=1}^{m p} \lambda_{n}^{2}(\tau)=\sum_{n=1}^{p} \operatorname{Tr}\left(b_{n}^{2}+2 a_{n} a_{n}^{*}\right), \\
\sum_{n=1}^{p m} \lambda_{n}^{2}(\tau) \geqslant 2 p m|c|^{\frac{2}{p m}},
\end{gathered}
$$

where the identity (2.2) holds true iff $a_{n} a_{n}^{*}=|c|^{\frac{1}{p m}} I_{m}$ and $b_{n}=0$ for all $n \in \mathbb{Z}$.

ii) Each determinant $D_{p}(z, \tau)=\operatorname{det}\left(M_{p}(z)-\tau I_{2 m}\right),(z, \tau) \in \mathbb{C} \times \mathbb{S}^{1}$ satisfies

$$
\begin{aligned}
D_{p}(z, \tau)=\prod_{j=1}^{2 m}\left(\tau_{j}(z)-\tau\right) & =c^{-1}(-\tau)^{m} \prod_{n=1}^{m p}\left(z-\lambda_{n}(\tau)\right) \\
& =c^{-1}(-\tau)^{m} \operatorname{det}\left(z I_{p m}-K_{p}(\tau)\right) .
\end{aligned}
$$

Proof. This lemma was proved in $\mathrm{KKu}$. For the reader's sake we recall the proof.

i) The identities (2.1) simply follow from (1.8) (also we use $\operatorname{Tr} a_{n} a_{n}^{*}=\operatorname{Tr} a_{n}^{*} a_{n}$ ).

Let $\varepsilon_{j, n}, j \in \mathbb{N}_{m}$ be eigenvalues of the matrix $a_{n} a_{n}^{*}, n \in \mathbb{N}_{p}$. Then (2.1) gives

$$
\begin{aligned}
\sum_{n=1}^{p m} \lambda_{n}^{2}(\tau) \geqslant \sum_{n=1}^{p} 2 \operatorname{Tr} a_{n} a_{n}^{*} & =2 \sum_{n=1}^{p m} \sum_{j=1}^{m} \varepsilon_{j, n} \geqslant 2 p m \prod_{j, n} \varepsilon_{j, n}^{\frac{1}{p m}} \\
& =2 p m\left(\operatorname{det} \prod_{1}^{p} a_{n} a_{n}^{*}\right)^{\frac{1}{p m}}=2 p m|c|^{\frac{2}{p m}} .
\end{aligned}
$$

Recall that the identity $\sum_{n=1}^{p} \sum_{j=1}^{m} \varepsilon_{j, n}=p m \prod_{j, n} \varepsilon_{j, n}^{\frac{1}{p m}}$ holds true iff each $\varepsilon_{j, n}=$ $\left(\prod_{j, n} \varepsilon_{j, n}\right)^{\frac{1}{p m}}=|c|^{\frac{2}{p m}},(j, n) \in \mathbb{N}_{m} \times \mathbb{N}_{p}$, that is $a_{n} a_{n}^{*}=|c|^{\frac{2}{p m}} I_{m}$ for all $n$. Also, $\sum_{n=1}^{p m} \lambda_{n}^{2}(\tau)=\sum_{n=1}^{p} 2 \operatorname{Tr} a_{n} a_{n}^{*}$ iff $b_{n}=0$ for all $n$ (see (2.1)).

ii) We fix $\tau \in \mathbb{S}^{1}$. Identity (1.2) yields $y_{n+1}=a_{n}^{-1}\left(\left(z-b_{n}\right) y_{n}-a_{n-1}^{*} y_{n-1}\right)$, which gives $\varphi_{p+1}=a_{p}^{-1} a_{p-1}^{-1} . . a_{1}^{-1} z^{p}+O\left(z^{p-1}\right), \varphi_{p}=O\left(z^{p-1}\right), \vartheta_{p+1}=O\left(z^{p-1}\right)$, 
$\vartheta_{p}=O\left(z^{p-1}\right)$ as $z \rightarrow+\infty$. The substitution of these asymptotics into (1.3) yields $D_{p}(z, \tau)=c^{-1}(-\tau)^{m} z^{p m}+O\left(z^{p m-1}\right)$ as $z \rightarrow \infty$, since eigenvalues of $M^{-1}(z)$ and $M^{*}(z), z \in \mathbb{R}$ coincide.

Let $K_{p}(\tau) f=\lambda(\tau) f$ for some eigenvalue $\lambda(\tau)$ and some eigenvector $f=\left(f_{n}\right)_{1}^{p} \in$ $\mathbb{C}^{p m}$. Then the definition of the matrix $M_{p}$ gives $M_{p}(\lambda(\tau))\left(f_{0}, f_{1}\right)^{\top}=\tau\left(f_{0}, f_{1}\right)^{\top}$, where $f_{0}=\tau^{-1} f_{p}$. Thus $\tau$ is an eigenvalue of $M_{p}(\lambda(\tau))$ and $\lambda(\tau)$ is a zero of $D_{p}(\cdot, \tau)$.

Firstly, let all eigenvalues $\lambda_{n}(\tau), n \in \mathbb{N}_{m p}$ of $K_{p}(\tau)$ be distinct. Then $\lambda_{n}(\tau), n \in$ $\mathbb{N}_{m p}$ are zeros of $D_{p}(\cdot, \tau)$, which yields (2.3) for all $z \in \mathbb{C}$, since the orders of the polynomials $\operatorname{det}\left(K_{p}(\tau)-z I_{m p}\right)$ and $D_{p}(z, \tau)$ coincide.

Secondly, consider the general case. Define the Jacobi operator $\mathcal{J}_{t}=\mathcal{J}+$ $t \operatorname{diag}\left(r_{n}\right)_{n \in \mathbb{Z}}, t \in \mathbb{R}$, where $r_{n}=n, n \in \mathbb{N}_{m p}$ and let $r_{n+p m}=r_{n}$ for all $n \in \mathbb{Z}$. Then the corresponding matrix $K_{p}(\tau, t)=K_{p}(\tau)+t \operatorname{diag}\left(r_{n}\right)_{n=1}^{p m}$, and let $D_{p}(z, \tau, t)$ be the corresponding determinant. Then all eigenvalues $\lambda_{n}(\tau, t)=t r_{n}+o(t), n \in \mathbb{N}_{m p}$ of $K_{p}(\tau, t)$ are distinct as $t \rightarrow \infty$ and in this case (2.3) holds true; i.e.,

$$
(-1)^{p m} \operatorname{det}\left(K_{p}(\tau, t)-z I_{p m}\right)=\frac{D_{p}(z, \tau, t)}{c^{-1}(-\tau)^{m}}=\prod_{n=1}^{p m}\left(z-\lambda_{n}(\tau, t)\right)
$$

for all $z \in \mathbb{C}$ and all large $t$. The functions in (2.4) are polynomials in $t$. Then the identities (2.4) hold true for all $t \in \mathbb{R}$, which yields (2.3), at $t=0$.

Lemma 2.2. For any $r \geqslant 0, s \geqslant 2$, the following identity holds true:

$$
\begin{aligned}
& \text { (2.5) } \sup _{x \in \mathcal{P}_{s}(r)} \sum_{1}^{s} x_{n}^{2}=2 s\left(\frac{r}{2}\right)^{\frac{2}{s}}, \\
& \mathcal{P}_{s}(r)=\left\{\left(x_{n}\right)_{1}^{s} \in \mathbb{R}^{s}: x_{1} \leqslant \cdots \leqslant x_{s}, \sum_{n=1}^{s} x_{n}=0, \prod_{n=1}^{s}\left|z-x_{n}\right| \leqslant r, \forall z \in\left[x_{1}, x_{s}\right]\right\} .
\end{aligned}
$$

Proof. Let $\|x\|^{2}=\sum x_{n}^{2}, x=\left(x_{n}\right)_{1}^{s} \in \mathbb{R}^{s}$. The set $\mathcal{P}_{s}(r)$ is compact. Then $\sup _{x \in \mathcal{P}_{s}(r)}\|x\|^{2}=\left\|x^{0}\right\|^{2}$ for some $x^{0}=\left(x_{n}^{0}\right)_{1}^{s} \in \mathcal{P}_{s}(r)$. Introduce a polynomial $p_{0}(z)=\prod_{n=1}^{s}\left(z-x_{n}^{0}\right)$. The polynomial $p_{0}^{\prime}$ has only real zeros $\widetilde{x}_{n}, n \in \mathbb{N}_{s-1}$. We will show that each $\left|p_{0}\left(\widetilde{x}_{n}\right)\right|=r, n \in \mathbb{N}_{s-1}$. Assume that there exist $1 \leqslant n_{1}<n_{2} \leqslant s$ such that

$x_{n_{1}-1}^{0}<x_{n_{1}}^{0} \leqslant x_{n_{2}}^{0}<x_{n_{2}+1}^{0}, \max _{z \in\left[x_{n_{1}}, x_{n_{2}}\right]}\left|p_{0}(z)\right|<r$, where $x_{0}^{0}=-\infty, x_{s+1}^{0}=+\infty$.

Introduce a polynomial $p_{\varepsilon}(z)=\prod_{n=1}^{s}\left(z-x_{n}^{\varepsilon}\right)$, where a vector $x^{\varepsilon}=\left(x_{n}^{\varepsilon}\right)_{n=1}^{s} \in \mathbb{R}^{s}$ is given by

$$
x_{n}^{\varepsilon}=x_{n}^{0}, n \neq n_{1}, n \neq n_{2} ; \quad \text { and } \quad x_{n_{1}}^{\varepsilon}=x_{n_{1}}^{0}-\varepsilon, x_{n_{2}}^{\varepsilon}=x_{n_{2}}^{0}+\varepsilon, \quad \varepsilon>0 .
$$

Using $x^{0} \in \mathcal{P}_{s}(r)$ and (2.6), we obtain

$$
x_{1}^{\varepsilon} \leqslant \cdots \leqslant x_{s}^{\varepsilon}, \quad \sum_{n=1}^{s} x_{n}^{\varepsilon}=\sum_{n=1}^{s} x_{n}^{0}=0, \quad \max _{z \in\left[x_{n_{1}}^{\varepsilon}, x_{n_{2}}^{\varepsilon}\right]}\left|p_{\varepsilon}(z)\right| \leqslant r,
$$

for sufficiently small $\varepsilon>0$. We rewrite $p_{\varepsilon}$ in the form

$$
p_{\varepsilon}(z)=p_{0}(z) g_{\varepsilon}(z), \quad g_{\varepsilon}(z)=\frac{\left(z-x_{n_{1}}^{\varepsilon}\right)\left(z-x_{n_{2}}^{\varepsilon}\right)}{\left(z-x_{n_{1}}^{0}\right)\left(z-x_{n_{2}}^{0}\right)}=\frac{\left(z-x_{n_{1}}^{0}+\varepsilon\right)\left(z-x_{n_{2}}^{0}-\varepsilon\right)}{\left(z-x_{n_{1}}^{0}\right)\left(z-x_{n_{2}}^{0}\right)} .
$$


Due to $x_{n_{1}}^{0} \leqslant x_{n_{2}}^{0}$ and $\varepsilon>0$ we deduce that $\left|g_{\varepsilon}(z)\right| \leqslant 1, z \in \mathbb{R} \backslash\left[x_{n_{1}}^{\varepsilon}, x_{n_{2}}^{\varepsilon}\right]$. Then (2.9) yields $\left|p_{\varepsilon}(z)\right| \leqslant\left|p_{0}(z)\right|, z \in \mathbb{R} \backslash\left[x_{n_{1}}^{\varepsilon}, x_{n_{2}}^{\varepsilon}\right]$ and thus (2.8) gives $\left|p_{\varepsilon}(z)\right| \leqslant r$, $z \in\left[x_{1}^{\varepsilon}, x_{s}^{\varepsilon}\right]$, and $x^{\varepsilon} \in \mathcal{P}_{s}(r)$ for all sufficiently small $\varepsilon>0$. We can obtain an estimate

$$
\left\|x^{\varepsilon}\right\|^{2}=\sum_{n=1}^{s}\left(x_{n}^{\varepsilon}\right)^{2}=\sum_{n=1}^{s}\left(x_{n}^{0}\right)^{2}+4 \varepsilon\left(x_{n_{2}}^{0}-x_{n_{1}}^{0}\right)+2 \varepsilon^{2}>\left\|x^{0}\right\|^{2},
$$

since $x_{n_{1}}^{0} \leqslant x_{n_{2}}^{0}$ and $\varepsilon>0$. But (2.10) and the condition $x^{\varepsilon} \in \mathcal{P}_{s}(r)$ contradict the identity $\sup _{x \in \mathcal{P}_{s}(r)}\|x\|^{2}=\left\|x^{0}\right\|^{2}$. Then the assumption (2.6) is not true and each $\left|p_{0}\left(\widetilde{x}_{n}\right)\right|=r, n \in \mathbb{N}_{s-1}$. Note that only polynomials $r \mathcal{T}_{s}(\alpha z+\beta), \alpha \in \mathbb{R} \backslash\{0\}$, $\beta \in \mathbb{R}$ (here $\mathcal{T}_{s}(\cos z)=\cos s z$ are the Tchebychev polynomials) have this property. Then $p_{0}(z)=r \mathcal{T}_{s}(\alpha z+\beta)$ for some $\alpha \in \mathbb{R} \backslash\{0\}, \beta \in \mathbb{R}$. We take $\alpha, \beta$ such that $p_{0}(z)=\prod_{n=1}^{s}\left(z-x_{n}^{0}\right), \sum_{n=1}^{s} x_{n}^{0}=0$ and then $p_{0}(z)=r \mathcal{T}_{s}\left(z r^{-\frac{1}{s}} 2^{\frac{1-s}{s}}\right)$, since (see [AS]

$\mathcal{T}_{s}(z)=\frac{1}{2} \sum_{k=0}^{\left[\frac{s}{2}\right]}(-1)^{k} \frac{s}{s-k} C_{k}^{s-k}(2 z)^{s-2 k}=2^{s-1} z^{s}-2^{s-3} s z^{s-2}+o\left(z^{s-2}\right) \quad$ as $z \rightarrow \infty$, where $C_{m}^{n}=\frac{n !}{m !(n-m) !}$. Then using the Viette formulas we get

$$
\begin{aligned}
\mathcal{T}_{s}(z) & =2^{s-1} \prod_{n=1}^{s}\left(z-z_{n}\right) \\
& =2^{s-1}\left(z^{s}-\xi z^{s-1}+\frac{1}{2}\left(\xi^{2}-\eta\right) z^{s-2}+o\left(z^{s-2}\right)\right) \quad \text { as } z \rightarrow \infty,
\end{aligned}
$$

where $z_{n}, n \in \mathbb{N}_{s}$ are zeroes of $\mathcal{T}_{s}$ and $\xi=\sum_{1}^{s} z_{n}, \eta=\sum_{1}^{s} z_{n}^{2}$. Then we get $\xi=0$ and $\eta=\frac{s}{2}$. This gives

$$
\sup _{x \in \mathcal{P}_{s}(r)}\|x\|^{2}=\left\|x^{0}\right\|^{2}=r^{\frac{2}{s}} 2^{\frac{2 s-2}{s}} \sum_{1}^{s} z_{n}^{2}=r^{\frac{2}{s}} 2^{\frac{2 s-2}{s}} \eta=2 s\left(\frac{r}{2}\right)^{\frac{2}{s}} .
$$

Proof of Theorem 1.1, i.a) The inclusion $\operatorname{Uni}_{p}\left(J^{0}\right) \subset \operatorname{Iso}_{p}\left(J^{0}\right)$ is obvious.

i.b) We prove that $\mathfrak{J}_{p}^{0} \subset \operatorname{Uni}\left(J^{0}\right)$. Let $J \in \mathfrak{J}_{p}^{0}$. Let the unitary operator $U=$ $\operatorname{diag}_{n \in \mathbb{Z}} u_{n}: \ell^{2}(\mathbb{Z})^{m} \rightarrow \ell^{2}(\mathbb{Z})^{m}$, where $u_{0}=I_{m} ; u_{n+1}=a_{n}^{*} u_{n}, n \geqslant 0 ; u_{n-1}=$ $a_{n-1} u_{n}, n \leqslant 0$. Then $U^{*} J U=J^{0}$ and $J \in \operatorname{Uni}_{p}\left(J^{0}\right)$.

i.c) Now we prove $\operatorname{Iso}_{p}\left(J^{0}\right) \subset \mathfrak{J}_{p}^{0}$, which finishes the proof of the theorem. Let $J^{1} \in \operatorname{Iso}_{p}\left(J^{0}\right)$ and let $\alpha, \beta \in \mathbb{R}$ be such that $|c|=\left|\prod a_{p} . . a_{1}\right|=1$ and $\sum_{1}^{p} \operatorname{Tr} b_{n}=0$ for the new operator $J=\alpha J^{1}+\beta$. Then $\left|\tau_{j}(z)\right|=1,(z, j) \in \sigma(J) \times \mathbb{N}_{2 m}$ for $J$, since $J^{1} \in \operatorname{Iso}_{p}\left(J^{0}\right)$. Consider the $p$-periodic operator $J$ as a $p k$-periodic operator for some $k \in \mathbb{N}$. Let $\lambda_{n, p k} \equiv \lambda_{n, p k}(1)$ be eigenvalues of $K_{p k}(1)$ (we write two indexes $n$ and $p k$, since we use eigenvalues for various $p$ (see after (1.8) )) and

$$
\lambda_{-} \equiv \lambda_{1, p k} \leqslant \lambda_{2, p k} \leqslant \cdots \leqslant \lambda_{+} \equiv \lambda_{p m k, p k} .
$$

The eigenvalues $\lambda_{ \pm}$belong to $\sigma(J)$. Then we have that $\left[\lambda_{-}, \lambda_{+}\right] \subset \sigma(J)$. Using $\left|\tau_{j}(z)\right|=1$ for all $(z, j) \in\left[\lambda_{-}, \lambda_{+}\right] \times \mathbb{N}_{2 m}$ and (2.3), we obtain

$$
\prod_{n=1}^{p m k}\left|z-\lambda_{n, p k}\right|=\left|D_{p k}(z, 1)\right|=\prod_{j=1}^{2 m}\left|1-\left(\tau_{j}\right)^{k}(z)\right| \leqslant \prod_{j=1}^{2 m}\left(1+\left|\tau_{j}\right|^{k}(z)\right) \leqslant 4^{m}
$$


for all $(z, j) \in\left[\lambda_{-}, \lambda_{+}\right] \times \mathbb{N}_{2 m}$, since $\left|\operatorname{det} \prod_{n=1}^{p k} a_{n}\right|=|c|^{k}=1$ and a sequence $a_{n}$ is $p$-periodic. A sequence $b_{n}$ is $p$-periodic. Then (2.1) yields

$$
\sum_{n=1}^{p m k} \lambda_{n, p k}=\sum_{n=1}^{p k} \operatorname{Tr} b_{n}=k \sum_{n=1}^{p} \operatorname{Tr} b_{n}=0 .
$$

The relations (2.11), (2.12), (2.13) and Lemma 2.2 give that

$$
\left(\lambda_{n, p k}\right)_{n=1}^{p m k} \in \mathcal{P}_{p m k}\left(4^{m}\right), \quad \sum_{n=1}^{p m k} \lambda_{n, p k}^{2} \leqslant 2 p m k C_{k}, \quad C_{k}=\left(\frac{4^{m}}{2}\right)^{\frac{2}{p m k}} .
$$

Then using (2.1) and $a_{n}=a_{n+p}, b_{n}=b_{n+p}$ for all $n \in \mathbb{Z}$, we obtain

$$
\sum_{n=1}^{p m k} \lambda_{n, p k}^{2}=\sum_{n=1}^{p k} \operatorname{Tr}\left(b_{n}^{2}+2 a_{n} a_{n}^{*}\right)=k \sum_{n=1}^{p} \operatorname{Tr}\left(b_{n}^{2}+2 a_{n} a_{n}^{*}\right)=k \sum_{n=1}^{p m} \lambda_{n, p}^{2} \leqslant k(2 p m) C_{k},
$$

which yields $\sum_{n=1}^{p} \lambda_{n, p}^{2} \leqslant 2 p m$, since we take $k \rightarrow+\infty$. Thus Lemma 2.1 implies $a_{n} a_{n}^{*}=I_{m}$ and $b_{n}=0$ for all $n \in \mathbb{Z}$, i.e. $J \in \mathfrak{J}_{p}^{0}$. By a), b) we have $\sigma(J)=[-2,2]=$ $[-2 \alpha+\beta, 2 \alpha+\beta]$, which yields $\alpha=1$ and $\beta=0$, i.e. $J^{1}=J \in \mathfrak{J}_{p}^{0}$.

ii) Using i), we deduce $J \in \mathfrak{J}_{p}^{0}$. The matrices $a_{n}, n \in \mathbb{Z}$ are unitary and they have only positive eigenvalues. Then all eigenvalues of $a_{n}, n \in \mathbb{Z}$ are equal to 1 , i.e. $a_{n}=I_{m}$ for all $n$. Then $J=J^{0}$.

Lemma 2.3. Let $a_{n}>0, b_{n}, n \in \mathbb{Z}$ be real matrices.

i) Let $\lambda$ be an eigenvalue of $K_{p}(\tau)$ and have multiplicity $m$ for some $\tau \in \mathbb{S}^{1} \backslash\{-1,1\}$. Then the multipliers have the form $\tau_{j}(\lambda)=\tau, \tau_{j+m}(\lambda)=\tau^{-1}$ for all $j \in \mathbb{N}_{m}$.

ii) Let $\lambda$ be an eigenvalue of $K_{p}(\tau)$ and have multiplicity $2 m$ for some $\tau \in\{-1,1\}$. Then each $\tau_{j}(\lambda)=\tau, j \in \mathbb{N}_{2 m}$.

Proof. i) The matrix $K_{p}(\tau)$ is self-adjoint. Let $K_{p}(\tau) f^{k}=\lambda f^{k}$ for some orthogonal eigenvectors $f^{k}=\left(f_{n}^{k}\right)_{n=1}^{m p}, k \in \mathbb{N}_{m}$. If $f_{0}^{k}=\tau^{-1} f_{p}^{k}$, then the definition of the matrix $M_{p}$ gives $M_{p}(\lambda)\left(f_{0}^{k}, f_{1}^{k}\right)^{\top}=\tau\left(f_{0}^{k}, f_{1}^{k}\right)^{\top}$. Note that $\widetilde{f}^{k}=\left(f_{0}^{k}, f_{1}^{k}\right)^{\top}$ define other components of the vector $f^{k}$, since $K_{p}(\tau)$ has special form; see (1.8). Then the vectors $\widetilde{f}^{k}, k \in \mathbb{N}_{m}$, are linearly independent vectors since $f^{k}$ are linearly independent vectors. Then $\tau$ has multiplicity at least $m$. The matrix $M_{p}$ is symplectic. Then $\frac{1}{\tau}$ is an eigenvalue of $M_{p}$ and $\frac{1}{\tau}$ has multiplicity at least $m$. We obtain the first statement, since $M_{p}$ is a $2 m \times 2 m$ matrix. The proof of ii) is similar.

Proof of Theorem 1.2. The statement i) follows from Lemma 2.1.

ii) Sufficiency. Recall that if $m=1$ and $J=J^{0}$, then $\operatorname{det}\left(M_{p}-\tau I_{2}\right)=\tau\left(\tau+\frac{1}{\tau}-\right.$ $\left.2 \mathcal{T}_{p}\left(\frac{z}{2}\right)\right)$, where $\mathcal{T}_{p}(z)=\cos (p \arccos z)$ is the Tchebychev polynomial. Moreover, the zeros of the polynomial $\mathcal{T}_{p}(z)-\cos \varkappa_{1}$ are given by $\lambda_{s}\left(e^{i \varkappa_{1}}\right)=2 \cos \frac{\varkappa_{1}+2 \pi(s-1)}{p}, s \in$ $\mathbb{N}_{p}$.

Thus if $J=J^{0}, m \geqslant 2$, then the corresponding monodromy operators $M_{p}$ satisfy $\operatorname{det}\left(M_{p}-\tau I_{2 m}\right)=\tau^{m} \prod_{j=1}^{m}\left(\tau+\tau^{-1}-2 \mathcal{T}_{p}(z 2)\right)$, which yields at $\tau=e^{i \varkappa_{1}}$,

$$
\operatorname{det}\left(M_{p}-e^{i \varkappa_{1}} I_{2 m}\right)=e^{i m \varkappa_{1}} 2^{m}\left(\cos \varkappa_{1}-\mathcal{T}_{p}(z / 2)\right)^{m} .
$$

This implies $\lambda_{s}\left(e^{i \varkappa_{1}}\right)=2 \cos \frac{\varkappa_{1}+2 \pi(s-1)}{p}$ for all $s \in \mathbb{N}_{m p}$.

Necessity. Let $\lambda_{s}\left(e^{i \varkappa_{1}}\right)=2 \cos \frac{\varkappa_{1}+2 \pi(s-1)}{p}$ for all $s \in \mathbb{N}_{m p}$. Then a direct calculation implies $\sum_{n=1}^{m p} \lambda_{n}^{2}\left(e^{i \varkappa_{1}}\right)=2 p m$, and the statement i) gives $J=J^{0}$. 
iii) The sufficiency is proved similarly to the case ii).

Necessity. We consider the case $e^{2 i \varkappa_{2}} \neq 1$; the proof of the case $e^{2 i \varkappa_{2}}=1$ is similar. Let $\lambda_{s}\left(e^{i \varkappa_{1}}\right)=2 \cos \frac{\varkappa_{1}+2 \pi(s-1)}{p}$ for all $s \in \mathbb{N}_{m p}$. Using (2.3), (2.15), we obtain

$$
\begin{aligned}
\prod_{j=1}^{2 m}\left(e^{i \varkappa_{1}}-\tau_{j}(z)\right) & =c^{-1}(-1)^{m} e^{i m \varkappa_{1}} \prod_{n=1}^{m p}\left(z-\lambda_{n}\left(e^{i \varkappa_{1}}\right)\right) \\
& =c^{-1}(-2)^{m} e^{i m \varkappa_{1}}\left(\mathcal{T}_{p}(z / 2)-\cos \varkappa_{1}\right)^{m},
\end{aligned}
$$

where $c=\operatorname{det} \prod_{n=1}^{p} a_{n}$. The eigenvalue $\lambda_{1}\left(e^{i \varkappa_{2}}\right)$ has multiplicity $m$. Then using Lemma 2.3 and substituting $z=\lambda_{1}\left(e^{i \varkappa_{2}}\right)=2 \cos \frac{\varkappa_{2}+2 \pi n_{1}}{p}$ and two multipliers $e^{ \pm i \varkappa_{2}}$ (given by Lemma 2.3) into (2.16), we obtain $\left(e^{i \varkappa_{1}}-e^{i \varkappa_{2}}\right)^{m}\left(e^{i \varkappa_{1}}-e^{-i \varkappa_{2}}\right)^{m}=$ $c^{-1} 2^{m} e^{i m \varkappa_{1}}\left(\cos \varkappa_{1}-\cos \varkappa_{2}\right)^{m}$, which yields $c=1$. Then the statement ii) gives $J=J^{0}$.

\section{ACKNOWLEDGMENTS}

The first author was partly supported by EPSRC grant EP/D054621 and DFG project BR691/23-1. Some parts of this paper were written at the Mathematical Institute of Humboldt University, Berlin. The second author is grateful to the institute for its hospitality.

\section{REFERENCES}

[AS] Abramowitz, M.; Stegun, A., eds. Handbook of Mathematical Functions with Formulas, Graphs, and Mathematical Tables. Dover Publications Inc., New York, 1992. MR.1225604 (94b:00012)

[BGGK] Bättig, D.; Grébert, B.; Guillot, J.-C.; Kappeler, T. Fibration of the phase space of the periodic Toda lattice. J. Math. Pures Appl. (9) 72 (1993), no. 6, 553-565. MR1249409 (95b:58073)

[BCK] Brüning, J.; Chelkak, D.; Korotyaev, E. Inverse spectral analysis for finite matrix-valued Jacobi operators, preprint, 2006.

[CG] Clark, S.; Gesztesy, F. On Weyl-Titchmarsh theory for singular finite difference Hamiltonian systems. J. Comput. Appl. Math. 171 (2004), 151-184. MR2077203 (2006i:39033)

[CGR] Clark, S.; Gesztesy, F.; Renger, W. Trace formulas and Borg-type theorems for matrixvalued Jacobi and Dirac finite difference operators. J. Differential Equations 219 (2005), no. 1, 144-182. MR2181033(2006e:47068)

[F] Flaschka, H. Discrete and periodic illustrations of some aspects of the inverse method, in Dynamical Systems, Theory and Applications, J. Moser (ed.), Lecture Notes In Physics, vol. 38, Springer, Berlin, 1975, pp. 441-466. MR0455033 (56:13274)

[GKM] Gesztesy, F.; Kiselev, A.; Makarov, K. A. Uniqueness results for matrix-valued Schrödinger, Jacobi, and Dirac-type operators. Math. Nachr. 239-240 (2002), 103-145. MR.1905666 (2003i:47047)

[K] Korotyaev, E. Gap-length mapping for periodic Jacobi matrices. Russ. J. Math. Phys. 13 (2006), no. 1, 64-69. MR2262812 (2007g:39028)

[KKr] Korotyaev, E.; Krasovsky, I. Spectral estimates for periodic Jacobi matrices. Commun. Math. Phys. 234 (2003), 517-532. MR1964381 (2003m:47056)

[KKu] Korotyaev, E.; Kutsenko, A. Lyapunov functions for periodic matrix-valued Jacobi operators, to appear in "Spectral Theory of Differential Operators: M. Sh. Birman 80th Anniversary Collection", American Mathematical Society Translations - Series 2, vol. 225, Amer. Math. Soc., Providence, RI, 2009.

[KKu1] Korotyaev, E.; Kutsenko, A. Inverse problem for the discrete 1D Schrödinger operator with small periodic potentials. Commun. Math. Phys. 261 (2006), 673-692. MR2197543 (2006j:81061) 
[KKu2] Korotyaev, E.; Kutsenko, A. Marchenko-Ostrovski mappings for periodic Jacobi matrices. Russ. J. Math. Phys. 14 (2007), no. 4, 448-452. MR2366203 (2008m:47043)

[vM] van Moerbeke, P. The spectrum of Jacobi matrices. Invent. Math. 37 (1976), no. 1, 45-81. MR0650253 (58:31226)

[RS] Reed, M.; Simon, B. Methods of Modern Mathematical Physics, Vol. IV, Analysis of Operators, Academic Press, New York-London, 1978. MR0493421 (58:12429c)

[T] Teschl, G. Jacobi Operators and Completely Integrable Nonlinear Lattices, Mathematical Surveys and Monographs, vol. 72, Amer. Math. Soc., Providence, RI, 2000. MR.1711536 (2001b:39019)

School of Mathematics, Cardiff University, Senghennydd Road, Cardiff, CF24 4AG, United KingDOM

E-mail address: KorotyaevE@cf.ac.uk

Department of Mathematics, Saint Petersburg State University, Saint Petersburg, 199034, Russia

E-mail address: kucenkoa@rambler.ru 